\title{
An Analysis of $g$ Strain in the EPR of Two [2Fe-2S] Ferredoxins. Evidence for a Protein Rigidity Model
}

\author{
D. O. Hearshen, ${ }^{*}$ W. R. Hagen, R. H. Sands, H. J. Grande, $†$ H. L. Crespi, \\ I. C. Gunsalus, $\S$ and W. R. DunhaM"
}

\begin{abstract}
Biophysics Research Division, Institute of Science and Technology, The University of Michigan, Ann Arbor, Michigan 48109; †Laboratory of Biochemistry, Agricultural University, de Dreijen 11, 6703 BC Wageningen, The Netherlands; $¥$ Chemistry Division, Argonne National Laboratory, Argonne, Illinois, 60439; and \$Department of Biochemistry, University of Illinois, Urbana, Illinois 60801
\end{abstract}

Received January 13, 1986; revised April 1, 1986

Replacing current notions of a paramagnetic center in a metalloprotein as a single entity in vivo with the more realistic concept of an ensemble of spin systems, each uniquely disturbed by its own surrounding protein, leads to a rigorous description of the spectroscopic factor, $g$, as a random variable whose statistical properties contain information on the rigidity of the protein. Generation of a consistent set of accurate simulations of very lownoise, multifrequency $(3,9,15 \mathrm{GHz})$ EPR data from selected $S=\frac{1}{2}$ proteins has now been achieved. This consistency lends support to the physical and biological inferences drawn from such simulations. The spectral contribution of magnetic hyperfine line-broadening is minimized by studying the ${ }^{56} \mathrm{Fe}$ reconstituted [2Fe-2S] cluster in fully deuterated ferredoxin from Synechococcus lividus and the ${ }^{2} \mathrm{H}_{2} \mathrm{O}$ exchanged [2Fe-2S] ferredoxin from Pseudomonas putida. High-resolution Mössbauer data on oxidized and reduced ${ }^{57} \mathrm{Fe}$ reconstituted $S$. lividus ferredoxin are also presented. The oxidized spectrum shows that the inequivalence of the two ferric ions in a [2Fe-2S] cluster can be resolved as two Mössbauer lines. The complete absence of this splitting in the ferric lines of the reduced spectrum is definitive proof that the reducing electron always resides at the same $\mathrm{Fe}$ atom in frozen aqueous solutions. To explain the distributed nature of the paramagnetic site in the ferredoxins, three models are considered: (1) a multiplicity of EPR states; (2) external perturbations to the molecular Hamiltonian; (3) a distribution in the crystal field Hamiltonian parameters. The first model is discarded, the second is possible but difficult to verify, and the third model is shown to fit the data well. The latter comparison requires a correction to literature expressions for the $g$ and $A$ tensors in [2Fe-2S] clusters. Statistical analysis strongly suggests that the EPR of metalloproteins in its details is a reflection of protein structure that distributes its spatial coordinates, accommodating different levels of rigidity, the more flexible parts being located at the outside. (c) 1986 Academic Press, Inc.

\section{INTRODUCTION}

In two previous papers a general mathematical description ( 1 ) and an efficient numerical algorithm (2) were developed to describe $g$-strained EPR spectra in metalloproteins. We are now in a position to apply these tools to specific examples from

* Present address: Department of Diagnostic Radiology, Henry Ford Hospital, Detroit, 2799 W. Grand Blvd., Detroit, Mich. 48202.

'To whom correspondence should be addressed. 
different classes of metalloproteins. Here we analyze the two-iron ferredoxins from Synechococcus lividus and from the bacterium Pseudomonas putida, referred to as algal ferredoxin and putidaredoxin, respectively. Algal ferredoxin functions as an electron transfer protein in photosynthesis (3) while putidaredoxin acts in electron transfer in a multienzyme system that hydroxylates camphor (4). These proteins are paramagnetic in the reduced state and exhibit EPR spectra below $140 \mathrm{~K}$, characteristic of an effective $S=\frac{1}{2}$ system. A detailed examination of the linewidth of the multifrequency EPR spectra of these proteins reveals two contributions: a field independent linewidth due to unresolved nuclear hyperfine couplings, and a linewidth that is linearly dependent upon the magnetic field (or microwave frequency) (5). This second contribution has been postulated to arise from a distribution in the principal $g$ values (6) and has been labeled " $g$ strain."

These ferredoxins were selected for analysis for a number of reasons: (1) Their physical characteristics, including optical, EPR, PMR, ENDOR, Mössbauer, and magnetic susceptibility, have been determined $((7,8)$ and Refs. quoted therein) though as yet no single crystals have been grown for analysis by single crystal EPR or X-ray crystallography. (2) A number of models for the molecular Hamiltonian have been proposed $(5,9-12)$ to explain the physical data, including the observed $g$ values and linewidths of the EPR spectra. These models can be reexamined for consistency in the context of the new statistical model (1). (3) Since the EPR is characterized by $S=\frac{1}{2}$, a relatively small number of terms can contribute to the Hamiltonian. (4) Algal ferredoxin was grown in a completely deuterated medium and reconstituted with ${ }^{56} \mathrm{Fe}$, resulting in a $10 \%$ reduction of the EPR linewidth over the spectrum of the native protein (7), while putidaredoxin, grown in $\mathrm{H}_{2} \mathrm{O}$, was reconstituted with ${ }^{56} \mathrm{Fe}$ in ${ }^{2} \mathrm{H}_{2} \mathrm{O}$. Since the effect of the nuclear hyperfine coupling is minimal, these proteins provide EPR spectra whose appearances are dominated by $g$ strain. (5) Finally, these two proteins are each representative of a further division of the [2Fe-2S] ferredoxins into two subgroups. Putidaredoxin is a member of a group characterized by narrow linewidths and an almost axial EPR spectrum, with apparent $g$ values around 2.02, 1.93, 1.93, while algal ferredoxin is a member of a group characterized by broader linewidths and a rhombic EPR spectrum with apparent $g$ values, 2.05, 1.96, and 1.88.

The problem we are addressing has its origins in the failure of current EPR simulation programs to exactly fit data for [2Fe-2S] proteins. These computer programs, however, approach a reasonable fit for any single EPR spectrum. The fits presented here are mainly different because they fit simultaneously the spectra taken at several frequencies. To prove necessity for our formulation, we must argue against all alternatives and show that there is no feature of the spectra that is unexplained by the formulation. We emphasize that underlying the complexity of the computer simulations is a set of physical principles that are very different from those used currently. For example, the use of a single $g$ tensor to describe a spin system is not a part of this formulation.

The statistical theory of $g$ strain. The statistical theory of $g$ strain has been described in detail elsewhere $(l)$. We summarize the salient features of the theory here. (1) To explain the large values and field dependence of the EPR linewidths, we hypothesize that the effective $g$ value is a distributed quantity. (2) The anisotropic nature of the linewidths suggests that the effective $g$ value is a function of other random variables, which we designate $\left\{p_{n}\right\}$. (3) The dimensionality of the set of $p$ random variables is 
three. (4) The elements of the $g$ tensor, from which the effective $g$ value is derived, depend linearly upon the $p$ random variables. The $3 \times 3$ matrix representation of the $g$ tensor, $\mathbf{g}$, thus depends linearly upon a "constant matrix", $\mathbf{g}^{0}$, a matrix, $\mathbf{P}$, whose elements are random variables, and the rotation that connects the $\mathbf{g}^{\mathbf{0}}$ and the $\mathbf{P}$ diagonal representations. (5) The observed linewidth is related to the variance of the effective $g$ value. (6) The distribution function of the $p$ random variables is joint normal.

The linewidth at a given powder orientation is thus a function of the three principal $g^{0}$ values, the first and second moments of the three $p$ random variables, and the rotation between $\mathbf{g}^{0}$ and $\mathbf{P}$. The first moments of the $p$ variables have been absorbed into the definition of $\mathbf{g}^{0}$, since this is mathematically equivalent to the case of nonzero first moments and a redefined $\mathbf{g}^{0}$. This was done for convenience, however, the two alternatives have different physical interpretations.

\section{MATERIALS AND METHODS}

The isolation of fully deuterated algal ferredoxin and of putidaredoxin has been described elsewhere $(13,14)$, as has the efficiency of the exchange of the ferredoxins with ${ }^{2} \mathrm{H}_{2} \mathrm{O}(15)$.

EPR spectra were taken under nonsaturating conditions at $\mathrm{X}$ band $(9 \mathrm{GHz})$ using a commercial Varian $E$ series spectrometer, at $\mathrm{S}$ band $(3 \mathrm{GHz})$ using a homemade spectrometer with a quartz-filled cavity, and at $P$ band $(15 \mathrm{GHz})$ using a homemade spectrometer with a cylindrical air-filled cavity. The characteristics of the $S$ and $P$ band spectrometers have been described elsewhere (16). The $\mathrm{S}$ and $\mathrm{P}$ band cavities were constructed to accommodate X-band size sample tubes, so that the same sample could be run at all three frequencies, thus eliminating possible sample-dependent variation in the data.

A previous paper describes our algorithm for $g$ strain (2). As has been done before $(11,12,17,18)$ we convolve the $g$-strain lineshape with another Gaussian. This "residual broadening" not only functions as the zero-width protector (2) but also is used to account for unresolved superhyperfine broadening in low-frequency spectra.

The minimization routines used in the computer calculations have been discussed elsewhere by Powell (19). These routines use simultaneous minimization of $N$ variables based on Powell's algorithm to determine the maximum-slope direction in the $N$ dimensional space of the minimized function and include an algorithm due to Brent (20) to search for the minimum along the current direction in $N$ space.

Mössbauer spectroscopic studies were performed on a homebuilt machine using published (21) data reduction procedures.

\section{RESULTS}

Multifrequency EPR data. Shown in Figs. 1 and 2 are the EPR spectra of algal ferredoxin and putidaredoxin taken at 3,9, and $15 \mathrm{GHz}$. These spectra have been plotted on the same $g$-value scale to emphasize their similarities. The bottom spectrum in Fig. 1 is slightly broader, demonstrating that there are still some unresolved superhyperfine interactions present, due to nearby ${ }^{14} \mathrm{~N}$ and, possibly, ${ }^{13} \mathrm{C}$ nuclei. This effect is even more pronounced in the $3 \mathrm{GHz}$ spectrum of putidaredoxin (Fig. 2) where there 


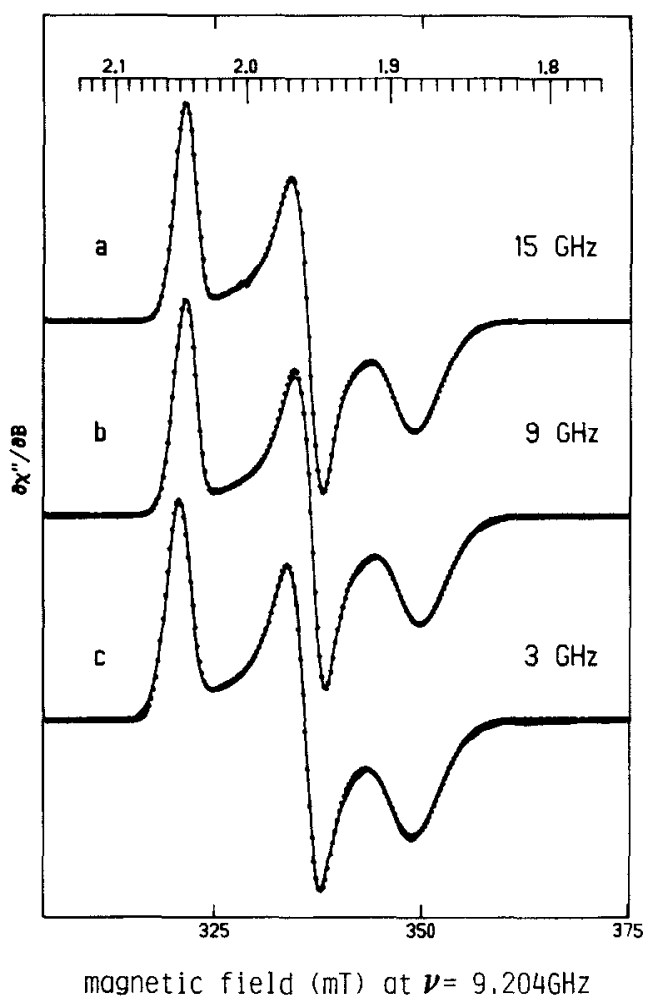

FIG. 1. Experimental (solid traces) and simulated (dotted traces) EPR spectra at three microwave frequencies of the fully deuterated, ${ }^{56} \mathrm{Fe}$ reconstituted, reduced [2Fe-2S] ferredoxin from Synechococcus lividus. See Table 1 for simulation parameters. EPR conditions for traces a, b, c, respectively: microwave frequencies, 14975, 9204, $3058 \mathrm{MHz}$; microwave powers, $0.12,4,10 \mathrm{~mW}$; modulation amplitudes, $0.6,0.1,0.4 \mathrm{mT}$; modulation frequency, $100 \mathrm{kHz}$; temperatures, $20,22,22 \mathrm{~K}$.

are proton hyperfine interactions present. In this case, the broadening due to unresolved hyperfine interactions at $3 \mathrm{GHz}$ is comparable to the $g$-strain linewidth.

The apparent linewidths of the principal $g$-value regions of the $9 \mathrm{GHz}$ spectrum are approximately 0.9-2.2 mT (millitesla). From the ENDOR spectra of ${ }^{1} \mathrm{H}$-algal ferredoxin, Anderson $e t$ al. have estimated the linewidth in magnetic field units from $T_{2}$, the transverse relaxation time, to be about $0.015 \mathrm{mT} \mathrm{(7),} \mathrm{some} \mathrm{two} \mathrm{orders} \mathrm{of}$ magnitude smaller than the observed linewidths. Thus the model for the observed lineshape cannot be based on a relaxation mechanism.

Spectral synthesis: Algal ferredoxin. The simulations for algal ferredoxin are also shown in Fig. 1. The final fits were obtained using an unweighted $\psi^{2}$ function (2I) in Fourier space, with $\psi^{2}$ between one and two for the 9 and $15 \mathrm{GHz}$ spectra, with a slightly higher $\psi^{2}$ at $3 \mathrm{GHz}$, due to an increase in the noise level. The spectral parameters, including the correlation coefficients and the rotation between the $p$ tensor principal axis system and the $g^{0}$ tensor principal axis system ( $l$ ) are given in Table 1 . The assignment of the $p$ variables as $p_{x}, p_{y}, p_{z}$ is ambiguous for a nonzero rotation. The linewidth function and consequently the EPR simulation are invariant under the 


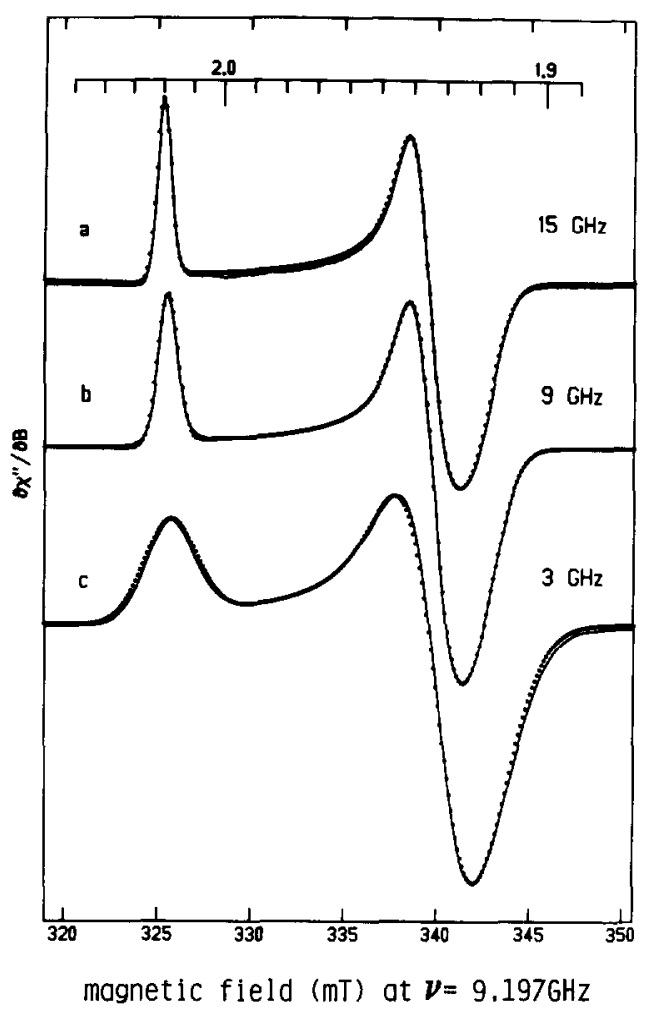

FIG. 2. Experimental (solid traces) and simulated (dotted traces) EPR spectra at three microwave frequencies of the ${ }^{2} \mathrm{H}_{2} \mathrm{O}$ exchanged, ${ }^{56} \mathrm{Fe}$ reconstituted, reduced [2Fe-2S] ferredoxin from Pseudomonas putida. See Table 2 for simulation parameters. EPR conditions for traces $\mathbf{a}, \mathbf{b}$, c, respectively: microwave frequencies, 14965, 9197, $3056 \mathrm{MHz}$; microwave powers, 0.39, 4, $10 \mathrm{~mW}$; modulation amplitudes, 0.2, 0.1, $0.2 \mathrm{mT}$; modulation frequency, $100 \mathrm{kHz}$; temperatures, $54,100,46 \mathrm{~K}$.

change of labels and rotations listed in Table 1 . Although we restrict the possible solutions to include only proper rotations, the linewidth function is also invariant to interchange of any two of the three signs of the off-diagonal matrix elements of the rotated P [cf. Eq. [34] of Ref. (1)] when there is full negative correlation. This means that all six possible permutations of the $p$ variables, each with its own rotation, are solutions to the EPR simulation problem. When the $p$ and $g^{0}$ tensors are not colinear, there is no longer a one-to-one correspondence between the apparent width of the spectrum and simulation linewidth parameters [cf. Eq. 16 in Ref. (1)].

The g-strain linewidth. Shown in Fig. 3 is a graph of the linewidth function versus orientation for deuterated algal ferredoxin. The distance from the origin to a point on the curve gives the value of the linewidth for a given orientation. Three projections are shown for rotations of the coordinate axes such that the $x-y, x-z$, and $y-z$ planes are each in the plane of the page. Figure 3 illustrates the differences between the present statistical model and other models for $g$ strain. Our model differs from previous models principally in the linewidth function. Contrast this function with those shown in Fig. 4. This figure shows a series of projections in the $x-z$ plane, using the $\sigma_{i}$ 's and 
TABLE 1

$g$-Strained EPR Simulation Parameters for the [2Fe-2S]

Ferredoxin from Synechococcus lividus

\begin{tabular}{|c|c|c|c|c|c|}
\hline \multicolumn{6}{|c|}{$g_{x}=1.881 \pm 0.003 ; g_{y}=1.958 \pm 0.003 ; g_{z}=2.050 \pm 0.003$} \\
\hline \multirow[b]{3}{*}{ No. ${ }^{a}$} & \multicolumn{5}{|c|}{$g$ Strain linewidth $^{b}$} \\
\hline & \multirow{2}{*}{\multicolumn{2}{|c|}{$\sigma(g$ units $) \times 10^{-3}$}} & \multicolumn{3}{|c|}{$\sigma(\mathbf{m T})$} \\
\hline & & & $3 \mathrm{GHz}$ & $9 \mathrm{GHz}$ & $15 \mathrm{GHz}$ \\
\hline 1 & & \pm 1.3 & 1.58 & 4.76 & 7.76 \\
\hline 2 & & \pm 1.6 & 0.31 & 0.94 & 1.53 \\
\hline 3 & & \pm 1.4 & 0.72 & 2.17 & 3.53 \\
\hline \multicolumn{6}{|c|}{$r_{12}=0.95 \pm 0.05 ; r_{13}=-0.95 \pm 0.05 ; r_{23}=-0.95 \pm 0.05$} \\
\hline \multicolumn{3}{|c|}{ Permutation ${ }^{c}$} & \multicolumn{3}{|c|}{ Rotation ( \pm 2$)$} \\
\hline 1 & 2 & 3 & $\alpha$ & $\beta$ & $\gamma$ \\
\hline$x$ & $y$ & $z$ & 40. & -159 & 16. \\
\hline$z$ & $x$ & $y$ & -16 & 104 & 112. \\
\hline$y$ & $z$ & $x$ & 104. & 106. & -154 \\
\hline$x$ & $z$ & $y$ & 106. & 104. & 68. \\
\hline$y$ & $x$ & $z$ & -140 & 21. & 16. \\
\hline$z$ & $y$ & $x$ & -14 & 106. & -26 \\
\hline
\end{tabular}

Note. The average residual broadening is $0.3 \mathrm{mT}$. The uncertainty in the $g$ values is due to uncertainty in calibration of field dials and frequency meters.

a The numbers refer to the three $p$ variables.

${ }^{b}$ Halfwidth at half maximum of a Gaussian lineshape.

c The six permutations of the three $p$ variables are all solutions to the EPR simulation where $p$ variable 1 is identified as $p_{x}$, etc.

the rotation from Fig. 3, varying only the correlation coefficients. Figure 3 has full negative correlation with a nonzero rotation, while Fig. 4 shows three other cases of interest. Figure $4 a$ has correlation coefficients of \pm 0.7 . Correlation coefficients of absolute value less than 0.7 do not have statistical significance, and thus nothing can be implied about any relationship between the $p$ random variables. Figure $4 \mathrm{~b}$ has correlation coefficients of zero. Complete independence between the random variables implies zero correlation, but the converse is not true (22). Figure $4 \mathrm{c}$ has full positive correlation coefficients. Both full negative correlation (Fig. 3) and full positive correlation (Fig. 4c) imply that the $p$ variables are linear functions of a single random variable; however, they give very different pictures of the linewidth versus orientation. This can be explained as follows. Full negative correlation means that the values of the three $p$ variables for each element of the ensemble are related in a specific way: when the value of one $p$ variable is increased, the values of the other two $p$ variables are decreased. As a result, there exist orientations where the effective $g$-value shifts go 

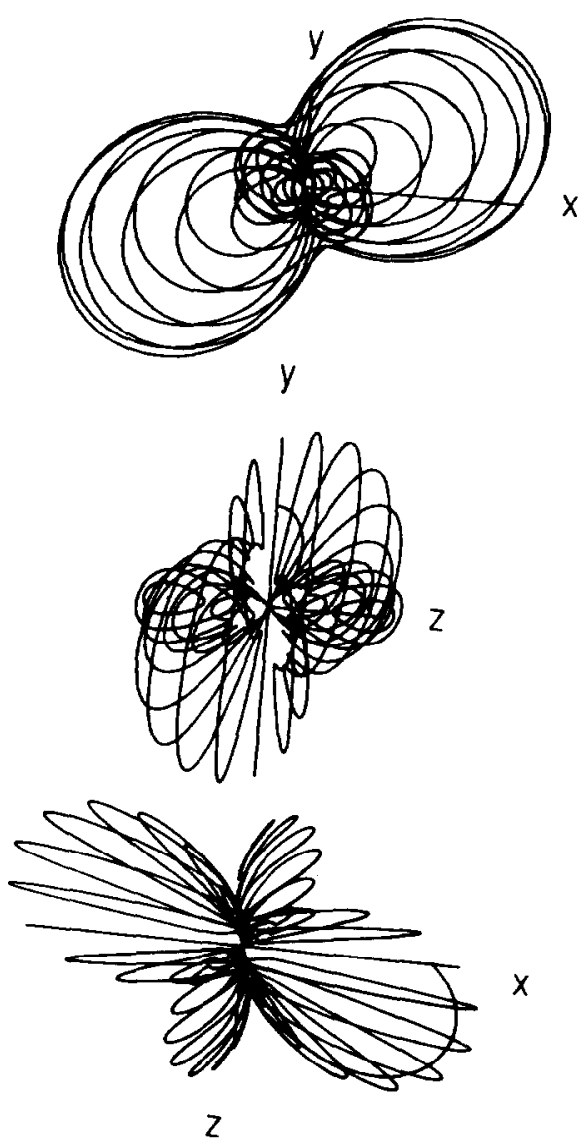

FIG. 3. Three projections of the linewidth function versus orientation for algal ferredoxin. The distance from the origin to a point on the curve represents the value of the linewidth for a given orientation. The values of $\sigma_{i}$ and the rotation can be found in Table 1 .

to zero, i.e. unstrained contributions to the powder spectra. The zero-width orientations are those for which the curve in Fig. 3 goes to the origin. On the other hand, full positive correlation implies that all three $p$ variables are being shifted in the same direction. The total $g$ shift can then never go to zero and the strain is always large. For completely uncorrelated shifts in the $p$ variables, a statistical distribution in the shift in $g$ can never go identically to zero. This is why Figs. $4 \mathrm{~b}$ and $\mathrm{c}$ are similar in appearance. The $g$-strain linewidth is very sensitive to departure from full negative correlation. If the absolute values of the correlation coefficients are reduced from 1 to 0.7 , the linewidth function (Fig. 4a) resembles the zero and full positive correlation cases more than the full negative correlation case.

The linewidth function used in the simulations shown in Fig. 1 included the residualbroadening term mentioned earlier. The value of the residual broadening was determined from the $3 \mathrm{GHz}$ simulation and was not a free parameter in the higher frequency simulations. Since the residual broadening is independent of magnetic field, its small 


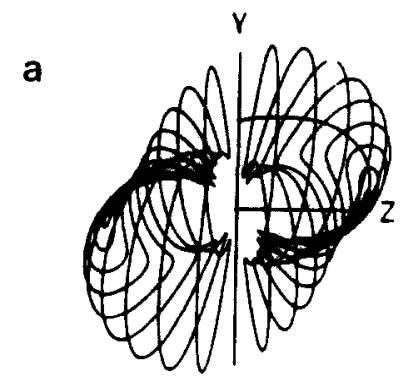

b
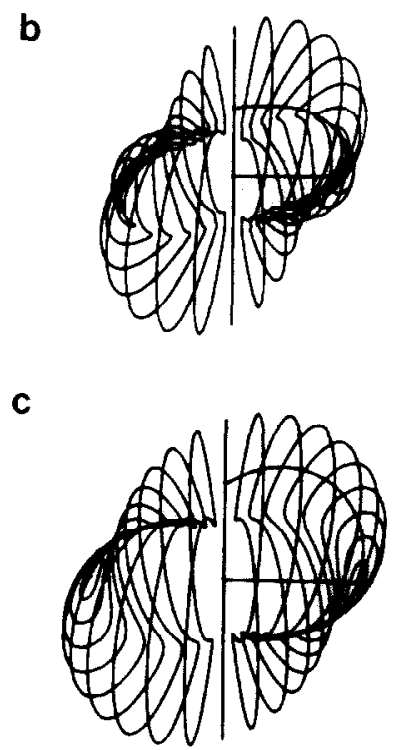

FIG. 4. The effect of correlation on the linewidth function. See text for details. All three correlation coefficients $r_{i j}$ are \pm 0.7 (trace a), 1 (trace b), 0 (trace c).

effect at $3 \mathrm{GHz}$ is virtually nonexistent at $15 \mathrm{GHz}$. We did not find it necessary to include a magnetic field-dependent linewidth term in our residual-linewidth expression as was done in the work of Hagen and Albracht (12).

Spectral synthesis: Putidaredoxin. The data and simulations for putidaredoxin are shown in Fig. 2, with the spectral parameters listed in Table 2. The goodness-of-fit parameter, $\psi^{2}$, is between 1 and 2 for the 9 and $15 \mathrm{GHz}$ spectra, and higher for the 3 $\mathrm{GHz}$ spectra even though the signal-to-noise ratio is about the same in all three spectra. The poorer fit at $3 \mathrm{GHz}$, especially in the $g_{z}$ region, illustrates the limits of the model for the residual broadening, i.e., the superhyperfine structure is not well approximated by a Gaussian distribution function. The actual fit was obtained with an anisotropic residual-width tensor modeled to be colinear with the $g$ tensor. This made a marginal improvement in the $3 \mathrm{GHz}$ spectrum and did not noticeably affect the higher frequency simulations. This relatively poor fit is not surprising, since the model for unresolved hyperfine interactions is being stretched when both the $g$-strain linewidth is relatively small (e.g., an order of magnitude difference at $g_{z}$ between algal ferredoxin and puti- 
TABLE 2

$g$-Strained EPR Simulation Parameters for the [2Fe-2S]

Ferredoxin from Pseudomonas putida

Fit A: Zero rotation, $\psi^{b}=1.45$

$g_{x}=1.921 \pm .0015 ; g_{y}=1.935 \pm .0015 ; g_{z}=2.020 \pm .0015$

$r_{12}=r_{13}=r_{23}=1$.

\begin{tabular}{lcccc}
\hline & \multicolumn{4}{c}{$g$ Strain linewidth $^{2}$} \\
\cline { 3 - 5 } & & \multicolumn{3}{c}{$\sigma(\mathrm{mT})$} \\
\cline { 3 - 5 } Var. & $\sigma(g$ units $) \times 10^{3}$ & $3 \mathrm{GHz}$ & $9 \mathrm{GHz}$ & $15 \mathrm{GHz}$ \\
\hline$x$ & $7.12 \pm 0.3$ & 0.42 & 1.27 & 2.12 \\
$y$ & $5.23 \pm 0.3$ & 0.30 & 0.92 & 1.54 \\
$z$ & $2.10 \pm 0.2$ & 0.12 & 0.36 & 0.59
\end{tabular}

Fit B: Nonzero rotation, $\psi^{2}=1.75$

$g_{x}=1.921 \pm .0015 ; g_{y}=1.935 \pm .0015 ; g_{z}=2.020 \pm .0015$

$r_{12}=r_{13}=-r_{23}=1$.

\begin{tabular}{|c|c|c|c|c|c|}
\hline \multirow[b]{3}{*}{ No. $^{a}$} & \multicolumn{5}{|c|}{$g$ Strain linewidth ${ }^{2}$} \\
\hline & \multirow{2}{*}{\multicolumn{2}{|c|}{$\sigma(g$ units $) \times 10^{3}$}} & \multicolumn{3}{|c|}{$\sigma(\mathrm{mT})$} \\
\hline & & & $3 \mathrm{GHz}$ & $9 \mathrm{GHz}$ & $15 \mathrm{GHz}$ \\
\hline 1 & \multicolumn{2}{|c|}{$8.81 \pm 0.3$} & 0.47 & 1.42 & 2.35 \\
\hline 2 & \multicolumn{2}{|c|}{$0.31 \pm 0.3$} & 0.02 & 0.06 & 0.10 \\
\hline 3 & \multicolumn{2}{|c|}{$7.91 \pm 0.3$} & 0.47 & 1.41 & 2.31 \\
\hline \multicolumn{3}{|c|}{ Permutation $^{3}$} & \multicolumn{3}{|c|}{ Rotation $\left( \pm 2^{\circ}\right)$} \\
\hline 1 & 2 & 3 & $\alpha$ & $\beta$ & $\gamma$ \\
\hline$x$ & $y$ & $z$ & -50 & 110. & 17. \\
\hline$z$ & $x$ & $y$ & 119. & 136. & 129. \\
\hline$y$ & $z$ & $x$ & -155. & 53. & 91. \\
\hline$x$ & $z$ & $y$ & -29 . & 136. & 51. \\
\hline$y$ & $x$ & $z$ & 140. & 110. & 163. \\
\hline$z$ & $y$ & $x$ & -115 & 53. & 89. \\
\hline
\end{tabular}

Note. The uncertainty in the $g$ values is due to uncertainty in calibration of field dials and frequency meters.

a The numbers refer to the three $p$ variables.

${ }^{b}$ Halfwidth at half maximum of a Gaussian lineshape.

$c$ The six permutations of the three $p$ variables are all solutions to the EPR simulation where $p$ variable 1 is identified as $p_{x}$, etc.

daredoxin) and the residual broadening is relatively large (fully deuterated algal vs ${ }^{2} \mathrm{H}_{2} \mathrm{O}$ exchanged putidaredoxin).

Shown in Fig. 5(L) is the linewidth function for putidaredoxin. At first glance, this picture is very different from the solution to algal ferredoxin. Not only is the rotation 

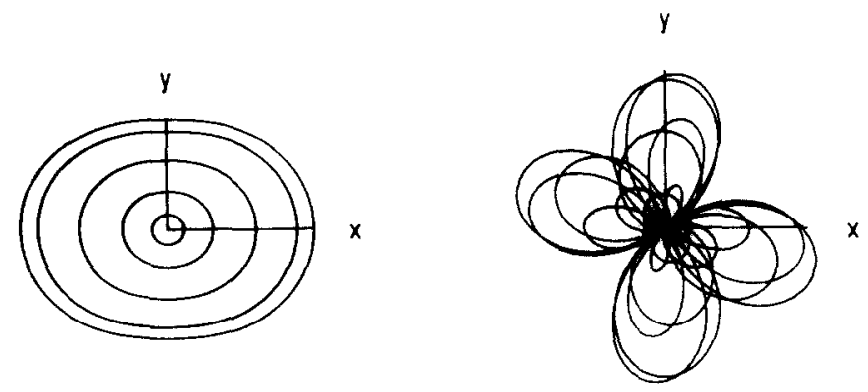

y
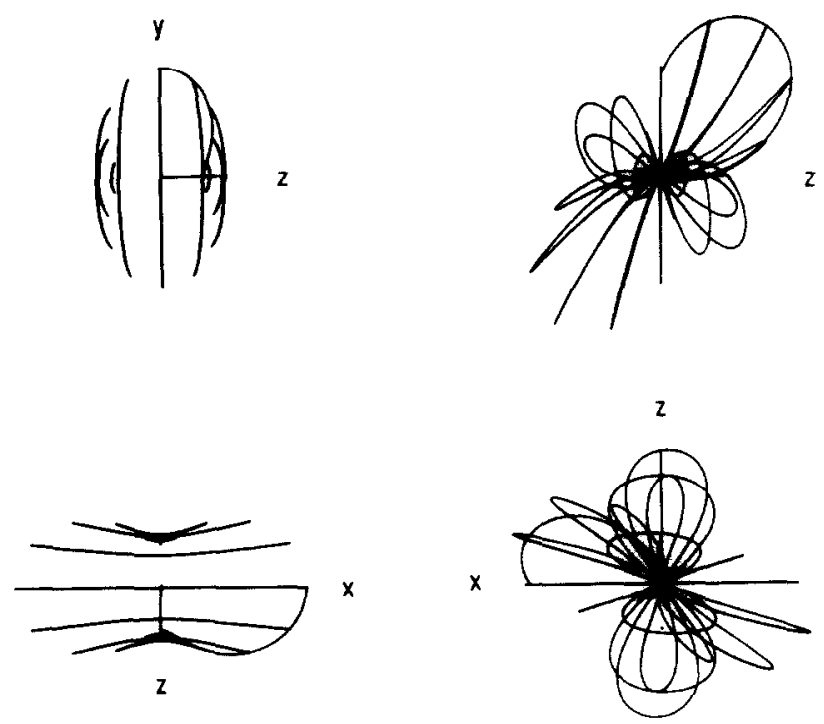

FIG. 5. Three projections of the linewidth function versus orientation for putidaredoxin. The left-hand set (L) represents the solution with zero rotation and full positive correlation corresponding to fit $A$ in Table 2. The right-hand set $(R)$ represents the solution with finite rotation and full negative correlation corresponding to fit $B$ in Table 2.

zero, i.e., the $g$ and $p$ tensors are colinear, but there is full positive correlation. In order to determine if this fit was unique, we tried to simulate the EPR spectrum with full negative correlation and a nonzero rotation by starting the minimization from a completely different set of spectral parameters. The result is given in Table 2 and in Fig. 5(R). The value of $\psi^{2}$ is slightly, but not significantly higher with full negative correlation and nonzero rotation. Because of the smaller strain and nearly axial spectrum in putidaredoxin, we are unable with these data to resolve any significant differences between the two cases of zero rotation plus positive correlation, and nonzero rotation plus negative correlation.

High-resolution Mössbauer spectra of algal ferredoxin. We have retaken the Mössbauer spectra of oxidized and reduced algal ferredoxin in order to increase the resolution of the resonances due to the two different iron atoms, by deconvolving the spectra from the source lineshape (21). The result, shown in Fig. 6, confirms a previous con- 


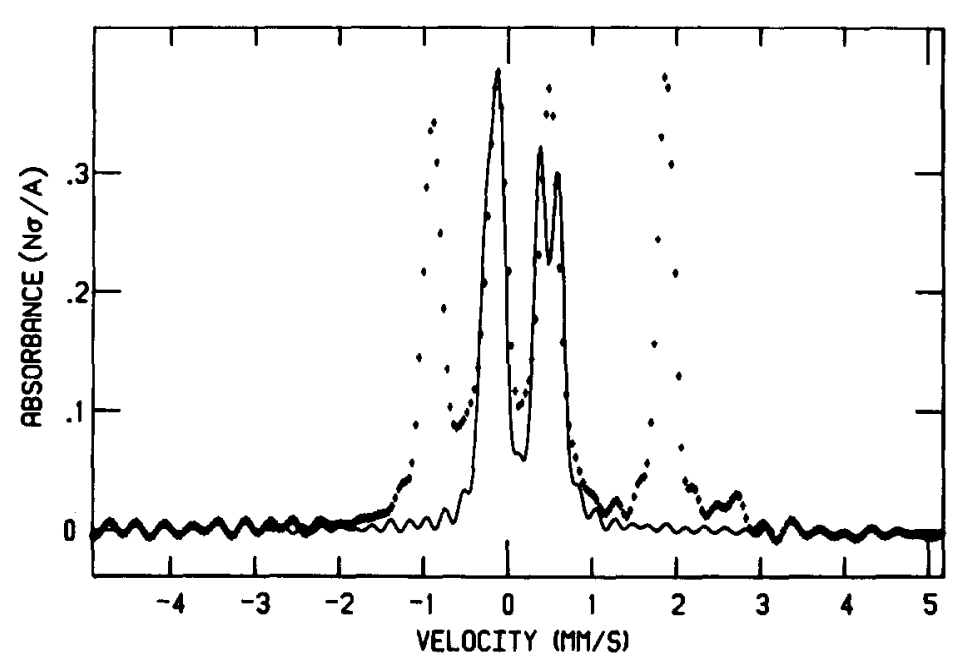

FIG. 6. High-resolution (i.e., source lineshape deconvolved) Mössbauer spectra of ${ }^{57} \mathrm{Fe}$ reconstituted [2Fe-2S] ferredoxin from Synechococcus lividus, taken at zero magnetic field. Solid curve: spectrum of oxidized ferredoxin taken at $\mathrm{T}=10 \mathrm{~K}$. Dotted curve: spectrum of reduced ferredoxin taken at $\mathrm{T}=175 \mathrm{~K}$. The height of the vertical bars is two standard deviations. $\sigma$ is the total cross section, $N / A$ is the areal density of ${ }^{57} \mathrm{Fe}$ for the reduced sample. The units on the ordinate refer to the spectrum of reduced ferredoxin. The amplitude of the spectrum of oxidized ferredoxin has been reduced by one-half for easy comparison. Isomer shifts are measured with respect to NBS-1541 iron metal at $\mathrm{T}=298 \mathrm{~K}$.

clusion (7) that the reducing electron always resides on one and the same iron atom (see below).

\section{DISCUSSION}

Several of the first explanations for the $g$ tensor of ferredoxins (23-25) turned out to be inconsistent with the assignment of spin and formal oxidation state to the iron atoms in reduced ferredoxins. Therefore, the cause of $g$ strain is not found in these models. At present, the possible models for $g$ strain fall into three categories: (1) those with a multiplicity of EPR states, (2) those with external perturbations to the molecular Hamiltonian, and (3) those with fluctuations of terms internal to the molecular Hamiltonian. We will now test these models against a combination of literature data and the new data presented above.

Model 1: Multiplicity of EPR states. In the derivation of the statistical model, the most obvious candidate for the identity of the $p$ variables is the $g$ tensor itself. In this case, Eq. [8] of Ref. (I) was used to define the linewidth function. The application to two-iron ferredoxins had mixed success. The linewidth function using this model successfully fits putidaredoxin at $15 \mathrm{GHz}$ and below (Fig. 2) but this model failed to fit algal ferredoxin (11). The initial extension of this model (11) was the proposed existence of two EPR states, each with three mean $g$ values and linewidths. These states, assumed to occur in a one-to-one ratio, were postulated to arise when the reducing electron (i.e., the electron that changes oxidized ferredoxin to reduced ferredoxin) has equal probability of being on either one of the iron atoms. While allowing a satisfactory fit to algal ferredoxin, this model suffers in comparison to the present 
result in that the uncertainty in the spectral parameters, especially the mean $g$ values of the two centers, is significantly larger than the uncertainty in the spectral parameters in Table 1. Also the number of free parameters in the two-state model is larger $(6 \mathrm{~g}$ values, 6 linewidths, 6 correlation coefficients, and the 1:1 ratio) than the present model ( $3 g$ values, 3 linewidths, 3 correlation coefficients, and 3 angles).

The main problem with the two-state model is that it is not supported by any other physical evidence. Both PMR (26) and Mössbauer data ((7) and Fig. 6) support the conclusion that there is a single electronic configuration in the reduced state. In the room temperature PMR spectra of reduced spinach ferredoxin, Poe et al. (26) recorded temperature-dependent resonance positions for several different lines. These resonance positions were interpreted by Dunham et al. (27) to be contact shifted resonances which were nonstandard due to the presence of the exchange interaction between the iron atoms in the molecule. The visible resonances in the PMR data were identified as those from $\alpha$-and $\beta$-carbon protons of the two cysteines coupled to the ferrous atoms. The positions of the resonances from the cysteinyl protons ligated to the ferric atom were also predicted (27) and later found near the indicated positions by Salmeen and Palmer (28). As a result of these investigations, it is possible to positively identify four of these resonances as due to the four protons from the two $\beta$-carbons of the two cysteine ligands to the ferrous atom. These resonance intensities integrated to single protons each (26). Therefore, one can say that either the reduced protein always has the same iron atom as the ferrous atom or there is a mixture of two protein structures, one with one iron atom reduced and one with the other reduced. These latter two conformations do not interconvert at a faster rate than $100 \mathrm{~Hz}$ or the lines from the ferrous and ferric shifted protons would begin to broaden. Furthermore, the two conformations must have identical proton environments at the ferrous site or there would be eight lines of intensity less than one proton each; hence the two-conformation idea is considered by us to be unlikely. We consider that it is far more likely that these four resonances are simply the result of four nonequivalent $\beta-\mathrm{CH}_{2}$ protons. The line positions are likely to be due mostly to contact shifts since Arison [data compiled in Ref. (29)] has shown that the proton exchange interactions are smaller than $0.2 \mathrm{ppm}$ for the three charge states of cysteine. The linewidth of these four resonances is on that order, whereas the differences in line positions is almost an order of magnitude larger. We therefore conclude that the room temperature PMR spectra of spinach ferredoxin show that there are four nonequivalent $\beta-\mathrm{CH}_{2}$ of the ferrous cysteines. Accordingly, the two-state model is incompatible with the room-temperature PMR measurements on the protein solutions.

In the frozen state, the proteins have been studied by Mössbauer spectroscopy. The two iron atoms have been shown to have inequivalent electronic environments from the Mössbauer spectra of oxidized putidaredoxin (30) and oxidized algal ferredoxin (7). These spectra exhibit the inequivalence in the unequal intensity and widths of two peaks, corresponding to two inequivalent quadrupole pairs. This inequivalence is actually resolved when the Mössbauer spectra are deconvolved with the source lineshape [following the methodology of (22)]. The resonances attributable to the ferric nucleus do not exhibit this inequivalence in the reduced spectrum. The deconvolved Mössbauer spectra of oxidized and reduced algal ferredoxin are plotted in Fig. 6 on the same velocity scan to emphasize the difference between the two ferric quad- 
rupole pairs in the oxidized state, and the inner two lines attributed to the ferric nucleus in the reduced state. These data support the conclusion that the reduced molecule has just one electronic configuration in the solid state, since the inner two lines in the deconvolved reduced-state spectrum are not broad enough to obscure the resolution of the two inequivalent ferric quadrupole pairs. The two-state model thus has little support other than the previous EPR simulations (11), which in fact have been improved by the present extension of the one-center statistical model.

Model 2: External perturbations to the molecular Hamiltonian. External perturbations have been postulated to give rise to the observed linewidths and lineshapes of two-iron ferredoxins $(5,12)$. At the heart of this work is the idea that the spin system at the active site of the protein is a unique physical system whose Zeeman interaction is describable by a Cartesian tensor. This physical system is stressed by an electric field (31) or mechanical force (12) that originates outside the protein. The relationship between the perturbing stress and the $g$ tensor of the spin system is described by another (stress-strain) tensor. While we have not done any experiment that relates to an external, physical cause of $g$ strain, our statistical analysis of the EPR spectra allows us to identify a conceptual ambiguity in this work. The $g$ tensor and stress-strain tensor should describe two unique physical systems: the internal or $g$ system and the connection between this inner system and the external world. However, it is presently impossible to identify this inner, unique system because its EPR spectra are characteristic of a distribution of systems. Furthermore, temperature-dependent X-ray crystallography indicates that protein crystals may also have a large distribution in conformational substates $(32,33)$. The mean square displacements of metmyoglobin atoms have been determined at $80 \mathrm{~K}, 220 \mathrm{~K}$, and $300 \mathrm{~K}$. Part of this displacement is attributed to conformational substates (33). Thus it is possible that the protein may undergo substantial structural change as a response to mechanical or electric stresses. If there is a structural distribution at the interior of the protein, then the identification of the random variables with the cause of the stress is greatly complicated since cause-effect relationships are difficult to distinguish when neither the solvent nor the solute molecules have a unique structure. The word "external" will have to be redefined so that the tensors used in the previous work have a possible physical interpretation.

Model 3: Fluctuations in the crystal-field Hamiltonian parameters. The observed $g$ values for a two-iron ferredoxin were successfully predicted first by an elementary crystal-field calculation (9). Antiferromagnetic coupling between the ferric atom, formally $S_{1}=\frac{5}{2}$, and the ferrous atom, formally $S_{2}=2$, was held responsible for the net total $S=\frac{1}{2}$ ground state. A previous investigation into possible sources of strain in this equation (5) considered the contributions from random processes varying the ferric and ferrous $g$ values separately. Variation in the ferric $g$ value is assumed to be smaller than variation in the ferrous [cf. Ref. (5)]. Variation in the ferric $g$ value is necessary since the elementary crystal-field calculation predicts no shift from the free electron $g$ value and hence no possible source of strain for the ferrous $g_{z}$ in this model. The strain from the ferrous and ferric $g$ values is assumed to be uncorrelated in order to explain the data. This is inconsistent with our results of full negative correlation. Furthermore, the assumptions in the crystal-field calculation of the ferrous $g$ tensor disallow any off-diagonal elements. This is inconsistent with the presence of a rotation between the $\mathbf{p}$ and $\mathbf{g}$ principal-axis systems. One logical extension of this model would 
be to include higher-order corrections in the ferrous $g$-tensor calculation. Even small off-diagonal elements in the ferrous $g$ tensor would produce the desired result. This extension has two possible parts. The first is a third order perturbation calculation of the $g$ shift. This calculation still does not allow any off-diagonal elements in the $g$ tensor (34). The second part of the extension is the addition of lower-symmetry distortions to the crystal-field potential. This can be further subdivided into distortions which can give rise to antisymmetric contributions to the $g$ tensor and distortions which give symmetric contributions to the $g$ tensor.

Model 3A: Asymmetric $g$ tensors. Asymmetric $g$ tensors have been shown to arise from anisotropic spin-orbit interactions and perturbations in the orbital wavefunctions and energies (35-37). Ham estimates that $g$ shifts due to the antisymmetric part of the $g$ tensor are only a few percent of the symmetric $g$ shifts, or when covalent bonding is strong, they may be comparable to the symmetric $g$ shift (37). Since in powder EPR we measure only the magnitude of the $g$ tensor, we cannot distinguish the antisymmetric shift from the symmetric part. Furthermore, we have calculated the effect of the antisymmetric contribution in the resonance condition to the renormalization of the probability density function (34) and found the EPR spectra to be unaffected for realistic sizes of the antisymmetric components. The presence of antisymmetric contributions to the $g$ tensor also does not agree with the results of our statistical model. Variation in the antisymmetric elements of the $g$ tensor without variation in the symmetric elements is not probable since both arise through changes in the crystal field. Even if variation in the antisymmetric elements alone were possible, the resulting variance in the effective $g$ value does not have the same functional form as the observed $g$-strain linewidth (34).

Model 3B: Symmetric variations in the crystal field. The active site of a two-iron ferredoxin in the reduced state consists of a high-spin ferric ion $\left({ }^{6} \mathrm{~S}\right)$ spin-coupled to a high-spin ferrous ion $\left({ }^{5} \mathrm{D}\right)$ to form an $S=\frac{1}{2}$ ground state. The ferric ion has no orbital angular momentum; therefore, it should have an isotropic $g$ tensor with a $g$ value around two. In fact, oxidized rubredoxin, which has high spin ferric ions in tetrahedral sulfur arrangements, has a $g$ tensor with values $2.03,1.98,2.09$ [computed from data in Ref. (38)] which shows itself at $g^{\prime} \simeq 4.3$ in EPR spectra for the middle doublet. The $A$ tensor for the ${ }^{6} \mathrm{~S}$ state should likewise be isotropic containing only the Fermi contact term; however, ENDOR measurements on putidaredoxin and algal ferredoxin have shown that this tensor is anisotropic: $18.5,20.7,15.2 \mathrm{MHz} A$ values for putidaredoxin; $19.2,18.2,14.6 \mathrm{MHz} A$ values for algal ferredoxin (8). The electricfield gradient at the ferric nucleus should have no contribution from the $3 \mathrm{~d}$ orbital occupancy, but some nonzero contribution from the ligands and the ferrous ion. Mössbauer spectroscopic studies ( 8 ) are consistent with this latter prediction. Overall, the idea that the ferric ion is a pure ${ }^{6} \mathrm{~S}$ state does not survive experimental testing. Since the $g$ and $A$ tensors are anisotropic, there are orbital components in the wavefunctions of the unpaired electrons of the ferric ion.

At the ferrous ion, the spin-orbit interaction is expected to lift some of the quenching of the orbital angular momentum. The spin Hamiltonian for high-spin ferrous ion is discussed by Varret (39). If we take from Varret's work the part relevant to distorted tetrahedral symmetry, we find that in his scheme there is a largest distortion, called "axial," and two kinds of smaller "rhombic" distortions. In what he calls "case 1", 
the rhombic change is aimed at two of the faces of the cube defining the tetrahedron in crystal-field theory. This is the more standard way of deforming the cube. The result is a small energy change in the $e$ set: $\left|x^{2}-y^{2}\right\rangle$ and $\left|z^{2}\right\rangle$, a larger splitting of $|x z\rangle$ and $|y z\rangle$ in the $t_{2}$ set with a smaller shift in the $|x y\rangle$ orbital position. In "case 2," the basic cube is rotated $45^{\circ}$ around the $z$ axis, so that the rhombic distortion is aimed at two of the edges of the cube. Accordingly, the $e$ set now must be renamed as: $\left|z^{2}\right\rangle$ and $|x y\rangle$, with $\left|x^{2}-y^{2}\right\rangle,|x z\rangle$ and $|y z\rangle$ in the energy elevated $t_{2}$ set. One of the terms in the rhombic distortion for both case 1 and case 2 mixes $\left|z^{2}\right\rangle$ with $\left|x^{2}-y^{2}\right\rangle$ so that the rhombically distorted ground state becomes

$$
\Psi_{0}=\cos \theta\left|z^{2}\right\rangle+\sin \theta\left|x^{2}-y^{2}\right\rangle
$$

The applicability of Varret's work to two-iron ferredoxins was seen by Bertrand and Gayda (10). They derived equations for the $g, A$, and EFG tensors under a case2 hypothesis and were able to show that the data for many ferredoxins fit this model very well. In the following arguments, we will show further corroboration of their model along with a few "refinements" that take into account our present work.

First, we would like to add to their paper the statement that high-spin ferrous ion is $3 \mathrm{~d}^{6}$ and, therefore, more than half-full. The Zeeman term is $\beta B(-L+2 S)(40)$, and there are likewise some sign changes in the operator equivalent in the magnetic hyperfine term. Accordingly, we derive the following equations for the $g$ and $A$ tensor principal components and the anisotropy, $\eta$, of the EFG tensor of the ferrous ion:

$$
\begin{aligned}
g_{2 x} & =g_{e}+\frac{8 \lambda}{\Delta_{y z}} \sin ^{2}(\theta+60) \\
g_{2 y} & =g_{e}+\frac{8 \lambda}{\Delta_{x z}} \sin ^{2}(\theta-60) \\
g_{2 z} & =g_{e}+\frac{8 \lambda}{\Delta_{x y}} \sin ^{2} \theta \\
A_{2 x} & =-P\left[\kappa-\frac{1}{7}\left[\frac{2\left(\Delta_{y z}-7 \lambda\right)}{\Delta_{y z}} \sin ^{2}(\theta+60)-1\right]\right] \\
A_{2 y} & =-P\left[\kappa-\frac{1}{7}\left[\frac{2\left(\Delta_{x z}-7 \lambda\right)}{\Delta_{x z}} \sin ^{2}(\theta-60)-1\right]\right] \\
A_{2 z} & =-P\left[\kappa-\frac{1}{7}\left[\frac{2\left(\Delta_{x y}-7 \lambda\right)}{\Delta_{x y}} \sin ^{2} \theta-1\right]\right] \\
\eta & =-\sqrt{3} \tan 2 \theta .
\end{aligned}
$$

These equations are correct for Varret's case 1 or case 2 . They are analogous to the equations in the previous work except that $\lambda$ is now positive and $A$ is now negative as it should be for a free, high-spin ferrous ion that is not spin-coupled to a ferric ion. For case- 1 systems, the $\left|x^{2}-y^{2}\right\rangle$ state is part of the $e$ set and thus is low lying in energy. The rhombic distortion can mix the $e$ set very strongly. The spin-orbit interaction will be to states in the $t_{2}$ set, and hence perturbation theory should be appropriate. 
For case 2 , the $\left|x^{2}-y^{2}\right\rangle$ mixing should be smaller than case 1 since $\left|x^{2}-y^{2}\right\rangle$ is now in the $t_{2}$ set. The spin-orbit interaction to the $|x y\rangle$ state should be much stronger than in case 1 due to the smallness on $\Delta_{x y}$. Perhaps perturbation theory will not be appropriate for the spin-orbit interaction in this case. If $\Delta_{x y}$ is less than a few hundred wave numbers, then $\lambda / \Delta_{x y}$ will not be small. Betrand and Gayda relied heavily on the $g$ values that were reported in the literature from either spectral simulations with "old" programs or by visual inspections of the turning points in the EPR spectra. Our work shows that neither of the two methods are reliable for two-iron ferredoxins. Furthermore, the Bertrand/Gayda model is based solely on the tensorial properties of the ferrous ion; whereas, the $g$ tensor of the ferric ion can have large rhombic components. Since $\left(\mathbf{S}_{1} \cdot \mathbf{S}\right) / S^{2}=\frac{7}{3}$ for the ferric site and $\left(\mathbf{S}_{2} \cdot \mathbf{S}\right) / S^{2}=-\frac{4}{3}$ at the ferrous site, the $g$ tensor anisotropies at the ferric site are "amplified" via spin coupling. Therefore, the $g$ values are not reliable numbers to judge the validity of this model. Instead, we will evaluate their model in terms of the $A$ tensor and anisotropy of the EFG tensor for the ferrous iron, which are measured by Mössbauer spectroscopy. We will also check whether their model predicts the covariance matrix $(1)$ for the EPR spectra of these proteins.

The anisotropy, $\eta$, in the EFG tensor of the ferrous ion is approximately zero for algal ferredoxin (7) and -3 for putidaredoxin (30). One can fit these numbers perfectly by having $\theta$ equal to zero for algal ferredoxin and $30^{\circ}$ for putidaredoxin. Thus, the model easily accounts for the differences in the EFG tensors, although no other model for these centers has been able to account for both of these EFG tensors. In Table 3, we compare the $A$-tensor values with those measured by ENDOR. We have divided the experimental numbers by $\left(\mathbf{S}_{2} \cdot \mathbf{S}\right) / S^{2}$ so that they will match the assumptions in Eqs. [2]-[4]. We have made assumptions concerning $P, \kappa, \lambda, \Delta_{x y}, \Delta_{x z}, \Delta_{y z}$ in line with our previous work (27) and also in line with those of Bertrand and Gayda (10). Specifically, $P=55 \mathrm{MHz}, \kappa=0.35, \lambda=80 \mathrm{~cm}^{-1}, \Delta_{x z}=6000 \mathrm{~cm}^{-1}, \Delta_{y z}=4000 \mathrm{~cm}^{-1}$, $\Delta_{x y}=600 \mathrm{~cm}^{-1}$. Note that two terms cancel in the equation for $A_{2 z}$ allowing a good fit to the previously unexplained fact that $A_{2 z}$ is approximately the same in both algal ferredoxin and putidaredoxin. The fit is admittedly less good for $A_{2 x}$ and $A_{2 y}$, but we are not compelled to abandon it for this reason because the model will allow a better fit at $A_{2 x}$ and $A_{2 y}$ with other values for $\theta, P, \kappa, \lambda, \Delta_{x z}$, and $\Delta_{y z}$ than these somewhat arbitrarily chosen values. Immediately, we can see that case 2 alone is compatible with the $A$-value data because $\Delta_{x y}$ must be around $600 \mathrm{~cm}^{-1}$ or $A_{2 z}$ will have a large dependence on $\theta$.

TABLE 3

Comparison of Theoretical (Eq. [3]) and Experimental Values for Ferrous $A$ Tensors

\begin{tabular}{lccc}
\hline & $A_{2 x}$ & $A_{2 y}$ & $A_{2 z}$ \\
\hline Algal ferredoxin: & -9.8 & -11.3 & -27.4 \\
$\quad$ Experimental theory $(\theta=0)$ & -17. & -16. & -27. \\
Putidaredoxin: & -13. & -18. & -26. \\
$\quad$ Experimental theory $(\theta=30)$ & -14. & -24. & -27. \\
\hline
\end{tabular}


As a measure of the dependence of the ferrous $g$ value on the parameter $\theta$, the independent variable in the Bertrand/Gayda model, the following expressions show the partial derivatives of these $g$ values in terms of $\theta$ :

$$
\begin{aligned}
& \frac{\delta g_{2 x}}{\delta \theta}=\frac{3 \lambda}{\Delta_{y z}} \sin (2 \theta+120) \\
& \frac{\delta g_{2 y}}{\delta \theta}=\frac{3 \lambda}{\Delta_{x z}} \sin (2 \theta-120) \\
& \frac{\delta g_{2 z}}{\delta \theta}=\frac{3 \lambda}{\Delta_{x y}} \sin 2 \theta .
\end{aligned}
$$

In Table 4, we evaluate these expressions at 0 and $30^{\circ}$, with the same assumptions used above for the parameter values. We have arbitrarily scaled the elements of the ${ }_{1} \mathbf{P}$ matrix to facilitate comparison in the table. When the linewidth parameters are written as a diagonalized ${ }_{1} P$ matrix [Eq. [32] in (I)] then only full correlation is allowed and full negative correlation is expressed by differing signs of its elements. Thus, both the data and Eq. [5] have the common quality that the correlation is full negative. In addition, the evaluations of Eq. [5] at 0 and $30^{\circ}$ show that the strain is two-dimensional, i.e., one of the partial derivatives is equal to zero. This trend is also present in the data, although much more clearly for putidaredoxin than for algal ferredoxin. Further inspection of Table 4, however, reveals the limitations of the Bertrand/Gayda model to predict $g$ strain under our interpretation: i.e., $\theta$ equal to 0 and $30^{\circ}$. For instance, the strain at the $z$ axis for putidaredoxin should be ten times that of the $y$ axis according to Table 4 , whereas the data show that these strains are approximately equal in magnitude. We feel that this lack of fit could be due to the presence of correlation between the value of $\theta$ and the values of the crystal-field splitting parameters. On the other hand, the unknowns presented by a possibly anisotropic ferric $g$ tensor could also explain the lack of fit. In particular, we note that the physical strain modes of the [2Fe-2S] core are joint displacements at both the ferric and ferrous cysteine sulfurs. Therefore, one may expect full correlation even if the ferric $g$ tensor is displaying strain effects. However, even if the ferric $g$ tensor is important to the $g$ strain, the

TABLE 4

Comparison of Theory (Eq. [5]) with the Linewidth Data ${ }^{a}$

\begin{tabular}{llll}
\hline & $x$ & $y$ & $z$ \\
\hline Algal ferredoxin: $\sigma$ from Table 1 times 6.3 & 0.15 & -0.08 & 0.03 \\
$\quad$ partial derivatives $(\theta=0)$ & 0.14 & -0.09 & 0.0 \\
Putidaredoxin: $\sigma$ from Table 2 times 60 & 0.02 & -0.47 & 0.53 \\
$\quad$ partial derivatives $(\theta=30)$ & 0.0 & -0.09 & 0.92 \\
\hline
\end{tabular}

${ }^{a}$ Linewidths are expressed as elements of the ${ }_{1} \mathbf{P}$ matrix [see Eq. [32] in Ref. (1)]. 
Bertrand/Gayda model remains as the only self-consistent explanation for the ferrous $A, g$, and EFG tensors in both algal ferredoxin and putidaredoxin.

\section{CONCLUSIONS}

The results of our work show that the $p$ tensor and $g$ tensor are not colinear. In terms of the Bertrand/Gayda model, this means that the $p$ tensor is diagonal along the same triad as the ferrous EFG tensor since the strain originates at the ferrous atom in this model, while the $g$ tensor principal axis system corresponds to neither of the $g$ tensors at the ferric and ferrous sites. In our treatment, the first moments of the $p$ system are added to the first moments of the $g$ distribution to form a redefined $g$ tensor. In general, the $g$ tensor formed in the above way is a full $3 \times 3$ matrix. After diagonalization, the principal axis $g$ values are defined. The Euler angles of our treatment define the relationship between this principal axis system and the $p$ tensor principal axis system; the orientations of the ferric $g$ tensor remain undetermined as do the $p$ tensor first moments. However, it is still possible to make several conclusions concerning the physical origin of $g$ strain since we have shown that rhombic strain must at least manifest itself at the ferrous iron.

It is possible that both $g$ strain and $g$-tensor anisotropy are freezing artifacts. If we reconsider the room temperature PMR data (26), we find that nonequivalence of the four lines attributable to the four $\beta-\mathrm{CH}_{2}$ protons at the ferrous ion implies that the local environment of the active center is sufficiently constant to maintain this inequivalence in solution. Note that the energy separations for these lines is much larger than can be attributed to exchange interactions of the protons themselves (29). Therefore, the lines are separated by differences in the Fermi contact interaction at the four protons, and there must be a rhombic component to the electron spin in room temperature solutions for the protein. Because the interior of the protein is stiff enough to maintain a rhombicity in solution, we can assume that this rhombicity is responsible for the $g$-tensor rhombicity in frozen aqueous solutions.

If the protein is very stiff in its interior, then we must also assume that it is less stiff or less homogeneous at its exterior in order to account for $g$ strain. Thus, the mechanical and electric stress tensor models may have valid interpretations. The question of whether $g$ strain results from the freezing process or from frozen-in-liquid conformers remains undetermined. However, Frauenfelder et al $(32,33)$ have found the presence of different conformers in single crystal samples. Apparently, a protein is able to crystallize while maintaining a distribution in conformeric states. Since our work implies that these conformeric differences consist of changes in the exterior of the molecule (as does the work of Frauenfelder $e t$ al.), these changes may account for the difficulties encountered in crystallizing the two-iron ferredoxins.

On the other hand, the work of Hagen $(12,18)$ has shown that changes in the freezing process can affect the EPR signal of many proteins. Freezing is also a process that can cause large local charge densities (41) and thereby change EPR spectra via electric fields (31). Therefore, if freezing is an important contribution to $g$ strain, then separating mechanical strain from electric-field strain will be a very difficult problem. A more intriguing question than the fundamental cause of $g$ strain is the question of the location of the protein groups whose structural changes allow the presence of $g$ strain. For example, what part of the protein can be assigned to the "stiff inner core?" 
The statistical model we have developed for EPR simulations may have wider applicability than to the class of metalloproteins that exhibit $g$ strain. Our interpretation of these results necessitates a revised conceptual framework for the analysis of EPR spectra of biomolecules. In particular, the idea that the EPR spectrum of a biomolecule can be described by a "single" effective spin Hamiltonian lacks the necessary precision to describe the apparent distribution in structures at the redox center. Our results and the results of the temperature-dependent X-ray crystallography mentioned earlier suggest that there is a distribution of protein conformations even in frozen solutions. If this distribution depends on more than one random variable and these variables are correlated, then the EPR spectra can appear very different from previous simulations. This possibility has both physical and biochemical consequences. Biochemical conclusions which rely heavily on interpretation of EPR spectra, especially the number and stoichiometry of paramagnets in complex proteins, will have to be reexamined in the context of the statistical model. Physical interpretations of EPR spectra, such as inferences of the spin state or the confirmation of the presence or absence of an interaction in the Hamiltonian, will also be affected. Negative correlation, which introduces "zero-width" orientations to the powder spectra, can result in a very skewed lineshape. Although we know of no experimental example, we have produced simulations based on a spin- $\frac{1}{2}$ Hamiltonian that mimics interaction spectra.

We are also considering the importance of the "zero-strain orientations" to the simulation of the ENDOR spectra from these proteins. In previous work $(6,7)$, we did not consider this possibility. It now appears that these orientations can greatly affect the ENDOR spectra taken where the EPR resonance is in the middle of the spectrum, i.e., in the area of the EPR spectrum where there are zero-strain orientations. We note that these are the ENDOR spectra whcre our simulation programs performed poorly compared to our fits where the EPR resonance was at the extremes of the spectra. Further work is necessary to establish the relationship between distributed protein structure and the fine details of ENDOR and high-spin EPR spectra.

\section{ACKNOWLEDGMENTS}

W. F. Filter assisted in the taking and subsequent reduction of Mössbauer data. W.R.H. gratefully acknowledges the award of a postdoctoral fellowship by the European Molecular Biology Organization. This work was supported in part by the Netherlands Organization for the Advancement of Pure Research (via a grant to H.J.G.) and by the U.S. Public Health Services via Grants GM 12176 and GM 32785, and by the Division of Chemical Sciences, Office of Basic Energy Sciences, U.S. Department of Energy.

\section{REFERENCES}

1. W. R. Hagen, D. O. Hearshen, R. H. Sands, AND W. R. Dunham, J. Magn. Reson. 61, 220 (1985).

2. W. R. Hagen, D. O. Hearshen, L. J. Harding, and W. R. DunhaM, J. Magn. Reson. 61, 233 (1985).

3. R. E. ANDERSON, Ph.D. thesis, University of Michigan, 1972.

4. I. C. Gunsalus, Hoppe-Seylers Z. Physiol. Chem. 349, 1610 (1968).

5. L. H. STRONG, Ph.D. thesis, University of Michigan, 1976.

6. J. Fritz, R. E. Anderson, J. A. Fee, G. Palmer, R. H. Sands, J. C. M. Tsibris, I. C. Gunsalus, W. H. ORME-JoHNSON, AND H. BEINERT, Biochim. Biophys. Acta 253, 110 (1971).

7. R. E. ANDERSON, W. R. DUNhaM, R. H. SANDS, A. J. BEARDEN, AND H. L. CRESPI, Biochim. Biophys. Acta 408, 306 (1975).

8. R. H. Sands AND W. R. DUNham, Q. Rev. Biophys. 7, 443 (1975). 
9. J. F. Gibson, D. O. Hall, J. H. M. ThoRnley, AND F. R. Whatley, Proc. Natl. Acad. Sci. USA 56, 987 (1966).

10. P. BERTRAND AND J.-P. GAYDA, Biochim. Biophys. Acta 579, 107 (1979).

11. D. O. Hearshen, W. R. Dunham, R. H. Sands, and H. J. Grande, in "Electron Transport and Oxygen Utilization" (Ho, C., Ed.), p. 395, Elsevier, New York, 1982.

12. W. R. Hagen and S. P. J. Albracht, Biochim. Biophys. Acta 702, 61 (1982).

13. H. L. CRESPI AND J. J. KaTZ, in "Methods in Enzymology" (C. H. W. Hirs and S. N. Timasheff, Eds.), Vol. 26, p. 627, Academic Press, New York, 1972.

14. D. W. Cushman, R. L. Tsai, AND I. C. Gunsalus, Biochem. Biophys. Res. Commun. 26, 577 (1967).

15. H. L. CRespi, A. G. KostKA, AND U. H. Smith, Biochem. Biophys. Res. Commun. 61, 1407 (1974).

16. R. C. Stevenson, Ph.D. thesis, University of Michigan, 1982.

17. W. Froncisz AND J. S. Hyde, J. Chem. Phys. 73, 3123 (1980).

18. W. R. HAGEN, J. Magn. Reson. 44, 447 (1981).

19. M. J. D. Powell, Comput. J. 7, 155 (1964).

20. R. BRENT, Ph.D. thesis, University of Michigan, 1971.

21. W. R. Dunham, C. T. WU, R. M. Polichar, R. H. Sands, and L. J. Harding, Nucl. Instrum. Methods 145, 537 (1977).

22. A. PAPoulis, "Probability, Random Variables, and Stochastic Processes," p. 266, McGraw-Hill, New York, 1965.

23. W. E. Blumberg and J. Peisach, in "Non-Heme Iron Proteins: Role in Energy Conversion" (A. San Pietro, Ed.), p. 101, Antioch Press, Yellow Springs, Ohio, 1965.

24. H. Brintzinger, G. Palmer, AND R. H. SANDS, Proc. Natl. Acad. Sci. USA 55, 397 (1966).

25. C. E. Johnson, R. C. Bray, R. CammaCk, and D. O. Hall, Proc. Natl. Acad. Sci. USA 63, 1234 (1969).

26. M. Poe, W. D. Phillips, J. D. Glickson, C. C. MCDonald, and A. San Pietro, Proc. Natl. Acad. Sci. USA 68, 68 (1971).

27. W. R. Dunham, G. Palmer, R. H. Sands, AND A. J. Bearden, Biochim. Biophys. Acta 253, 373 (1971).

28. I. Salmeen and G. Palmer, Arch. Biochem. Biophys. 150, 767 (1972).

29. G. C. K. Roberts AND O. JARDETzKY, in "Advances in Protein Chemistry" (C. B. Afinsen, J. T. Edsall, and F. M. Richards, Eds.), Vol. 24, p. 447, Academic Press, New York, 1970.

30. E. MUNCK, P. G. DebrunNer, J. C. M. Tsibris, AND I. C. Gunsalus, Biochemistry 11, 884 (1972).

31. W. B. Mims and J. PeISACH, Biochemistry 13, 3346 (1974).

32. H. Frauenfelder, G. A. Petsko, and D. Tsernoglou, Nature (London) 280, 558 (1979).

33. H. Hartmann, F. Parak, W. Steigemann, G. A. Petsko, D. Ringe Pontzi, and H. Frauenfelder. Proc. Natl. Acad. Sci. USA 79, 4967 (1982).

34. D. O. HEARSHEN, Ph.D. thesis, University of Michigan, 1983.

35. F. K. KNEUBUHL, Phys. Lett. 2, 163 (1962).

36. F. K. KNEUBUHL, Phys. Kondens. Mater. 1, 410 (1963).

37. F. S. HaM, J. Phys. Chem. Solids 24, 1165 (1963).

38. J. Peisach, W. E. Blumberg, E. T. LODE, AND M. J. COON, J. Biol. Chem. 246, 5877 (1971).

39. F. VARret, J. Phys. (Paris) 37, C6, 437 (1976).

40. J. S. GrifFITH, "The Theory of Transition-Metal Ions," p. 355, Cambridge Univ. Press, Cambridge, 1961.

41. R. J. CHENG, Science 170, 1395 (1970). 